\title{
PVU and wave-particle splitting schemes for Euler equations of gas dynamics
}

\author{
S M DESHPANDE, N BALAKRISHNAN and S V RAGHU- \\ RAMA RAO
}

CFD Laboratory, Department of Aerospace Engineering, Indian Institute of Science, Bangalore 560 012, India

\begin{abstract}
A new way of flux-splitting, termed as the wave-particle splitting is presented for developing upwind methods for solving Euler equations of gas dynamics. Based on this splitting, two new upwind methods termed as Acoustic Flux Vector Splitting (AFVS) and Acoustic Flux Difference Splitting (AFDS) methods are developed. A new Boltzmann scheme, which closely resembles the wave-particle splitting, is developed using the kinetic theory of gases. This method, termed as Peculiar Velocity based Upwind (PVU) method, uses the concept of peculiar velocity for upwinding. A special feature of all these methods is that the unidirectional and multidirectional parts of the flux vector are treated separately. Extensive computations done using these schemes demonstrate the soundness of the ideas.
\end{abstract}

Keywords. Upwind methods for Euler equations; wave-particle splitting; Boltzmann schemes; peculiar velocity based upwinding.

\section{Introduction}

Computational Fluid Dynamics (CFD) a new revolutionary tool in aerodynamic design and analysis, is progressing at a very fast pace and enormous developments in algorithm development, grid generation and postprocessing have taken palce in the last twenty years. CFD aims at solving numerically the partial differential equations of fluid dynamics and hence requires the use of digital computers which have progressed over years at a breathtaking pace. The ultimate aim is to obtain the direct numerical simulation of unsteady Navier-Stokes equation for an arbitrarily large Reynolds number for practical geometrical configurations such as aircraft, missile, launch vehicle, helicopters, ships or submarines. This aim has not been realised as yet in spite of the development of massively parallel computers and numerical solution of only some simple flow problems such as flow through a channel, flow past a flat plate, flow around a cylinder have been attempted using Direct Numerical Simulation of Turbulence (DNS). Even though it is true that the numerical solution of Navier-Stokes equation must be sought for every flow problem, there are many 
problems for which aerodynamic analysis and design can be done using lower level approximations which include potential flows governed by Laplace's or full potential equation, attached flows for which Euler equations are a valid approximation, thin layer Navier-Stokes equation (TLNS) and so on. In this paper we are going to concentrate on numerical methods for the solution of Euler equations of gas dynamics. Many problems arising in the aerodynamic design and analysis of the aerospace vehicles require the solution of Euler equations. Some notable examples are flow past delta wings, flow through ramjet type intakes, external supersonic flow past launch vehicles, hypersonic rentry flows. These eqautions also need to be solved in cases of flow problems where viscous effects are approximately taken into account by solving the well-known boundary layer equations which again require the inviscid solutions as the input. Further a good Euler solver is a prerequisite to the development of a good Navier-Stokes solver. Therefore constructing numerical schemes for solving the Euler equations has been one of the principal subjects of research among the CFD community for the last decade.

An ideal numerical scheme, none exists at all as of now, is the one which satisfies several requirements:

(1) It must be robust, that is, it should work for a wide variety of flow conditions covering all geometrical shapes encountered in practice with Mach number and angle of attack varying over a large range and various boundary conditions. Further, the code based on this method must work over different type of grids such as unstructured tetrahedral, structured hexahedral, clustered/unclustered and hybrid grids with the gric spect ratio and the skewness varying widely.

(2) The numerical schemes must be accurate enough to capture shocks, contact surfaces, shear layers with acceptable accuracy and resolution. (3) The scheme must be easy to code and be computationally inexpensive and must rapidly converge to the steady state whenever necessary.

(4) It must be adaptable to computer architecture in the sense that it should be vectorisable and should admit massive parallelism.

In summary, an ideal numerical method must be robust, efficient, accurate, rapidly converging and adaptable to new energing computer architecture. Unfortunately, in spite of several years of research in algorithm development, such an ideal scheme does not exist till date. The search for the elusive best scheme is on.

Several interesting characteristics have emerged about numerical schemes after several years of intensive research. First, it has been found that even if a numerical scheme is consistent and stable in the von Neumann sense it need not converge to the solution. Lax-Wendroff scheme and MacCormack scheme do require Total Variation Diminishing (TVD) fixes in order to supress pre-shock and post-shock wiggles encountered in capturing discontinuities. Without the TVD fix these and many other second order accurate schemes can cause violent oscillations in the flow variables leading to negative values of pressure and density. The mathematical theory of stability of numerical schemes for nonlinear partial differential equations together with a boundary condition treatment is just not available today to designers of numerical schemes to serve as guidelines for algorithm development. Secondly many first-order schemes possessing TVD property do not have solution reliability. For example Roe's method (Roe 1981) based on the approximate Riemann solver can admit unphysical shocks (called carbuncle shocks), may fail in capturing large rarefaction waves (Quirk 1992) and is known to converge to rotationally asymmetric solutions 
in case of supersonic flow past a hemisphere ( $\mathrm{P} \mathrm{K}$ Sinha, private communication). A lack of robustness has also been reported for the Osher method (Osher \& Solomon 1982 ) in capturing strong detached shock. Many fixes have been proposed to cure some of these failures, but these fixes are not universal and are known to destroy solution accuracy. Harten's entropy fix is known to spoil the high resolution property of Roe's scheme (Quirk 1992). Flux Vector Splitting methods on the other hand do possess solution reliability and capture shocks and large rarefaction waves without any problem but they are notorious for smearing the contact discontinuity (van Leer 1990). They also cause unacceptably large smearing of boundary layer (van Leer 1990). Central differencing schemes with artificial dissipation (Jameson et al. 1981) have their own problems. These schemes contain many tuning parameters which must be adjusted for robustness, accuracy and convergence. It has now become clear that having the correct amount of dissipation is the key to the design of an ideal numerical scheme. MacCormack (1990) has rightly observed it is all dissipation. Lastly, it has been found that a numerical scheme that solves a discrete mathematical model, which is an approximation to the partial differential equation purported to be solved, has adequate robustness if the discrete mathematical model mimics the physics of the flow as closely as possible. As an example to illustrate this principle we may cite the case of upwind methods which take into account the signal propogation property of the Euler equations by appropriately choosing the stencil of grid points. The Flux Vector Splitting schemes as noted above are very robust. One of the Flux Vector Splitting Schemes due to Deshpande \& Mandal (Deshpande 1986c, Mandal 1989, Mandal \& Deshpande 1993) called the Kinetic Flux Vector Splitting scheme (KFVS) has resulted in an extremely robust code BHEEMA routinely used at DRDL, Hyderabad for computing low speed, high speed and hypersonic flows around a variety of practical configurations. In the absence of clearcut and complete set of guiding principles to be used while constructing an ideal numerical method, we follow the following methodology while searching for new algorithms, (1) New directions and lines of research leading to novel algorithm development must be constantly explored. (2) Once these ideas take a concrete shape in the form of a scheme then it should be tested for robustness, accuracy, solution reliabillity, covergence property and adaptability by trying it on the largest set of fluid flow problems possible. (3) Suitable modifications in the algorithm should be progressively introduced for slowly but steadily marching towards the best scheme. We study in detail a new line of research called wave-particle splitting which is closely related to an allied idea termed Peculiar Velocity based Upwind (PVU) methods at the Boltzmann level.

\section{Wave-particle splitting}

\subsection{Acoustic flux vector splitting}

The Euler equations of gas dynamics can be written in the differential form of the conservation law as,

$$
\frac{\partial U}{\partial t}+\frac{\partial F}{\partial x}+\frac{\partial G}{\partial y}+\frac{\partial H}{\partial z}=0
$$


where $U=$ Conserved variable vector, $\mathbf{Q}=i_{x} F+i_{y} G+i_{z} H=$ flux vector and are defined by the relations,

$$
U=\left[\begin{array}{l}
\rho \\
\rho u \\
\rho v \\
\rho w \\
e
\end{array}\right] ; F=\left[\begin{array}{l}
\rho u \\
p+\rho u^{2} \\
\rho u v \\
\rho u w \\
(e+p) u
\end{array}\right] ; G=\left[\begin{array}{l}
\rho v \\
\rho v u \\
p+\rho v^{2} \\
\rho v w \\
(e+p) v
\end{array}\right] ; H=\left[\begin{array}{l}
\rho w \\
\rho u w \\
\rho v w \\
p+\rho w^{2} \\
(e+p) w
\end{array}\right]
$$

Here $\rho=$ mass density, $u, v, w$ are the components of velocity along the $x, y, z$ directions, $p=$ pressure and $e=$ specific total energy per unit volume and is given by,

$$
e=\frac{p}{(\gamma-1)}+\frac{1}{2} \rho\left(u^{2}+v^{2}+w^{2}\right)
$$

Even though in (2) $F, G, H$ are expressed as functions of the primitive variables $\rho, u, v, w, p$ they can also be expressed as functions of the conserved variables $U$. Equations (1) are nonlinear hyperbolic partial differential equations for the unknown $U$ and must be solved with suitable boundary conditions. Because of hyperbolicity these vector conservation laws involve propagation of waves which are the wellknown examples of nonlinear waves. Any numerical scheme if it claims to mimic the physics of the flow, must take into account the appropriate directions of information propagation. Currently there are two different ways of incorporating hyperbolicity into numerical schemes. The first one is the flux difference splitting where Riemann's initial value problen is solved approximately. Two important flux difference splitting schemes are currently available, one by Roe (1981) and the other by Osher (Osher \& Solomon 1982). The underlying physical model in these approaches is the interaction among cells through wave propagation. The variables are assumed to be constant within a cell and therefore fluid variables undergo jumps when we cross a cell face. These jumps are broken into waves using the Riemann solver and thus the cells interact with one another through waves. The second approach involves splitting of the flux vector into two parts $F^{+}, F^{-}$(for the $x$-component of $\mathbf{Q}$ ) such that the Jacobian $\partial F^{+} / \partial U$ has all positive eigenvalues and the Jacobian $\partial F^{-} / \partial U$ has all negative eigenvalues. The flux vector splitting of Steger \& Warming (1981), van Leer (1982) fall under this class. The underlying physical model here is the particle model. The flux vector splitting schemes can be regarded as a generalisation of the Courant-Isacson-Rees scheme or as a Beam scheme (Sanders \& Prendergast 1974) wherein it is tacitly assumed that there are in case of a 1-D problem two beams of particles moving in opposite directions. For this reason the FVS is also sometimes referred to as pseudo-particle method. The particle nature of FVS is even more obvious when we consider the KFVS method which is derived from the Boltzmann equation of kinetic theory of gases using the moment method strategy (Deshpande 1986c). An interesting question arises here as to whether we can construct new schemes using both the particle model and the wave model of fluid flow. There is a strong physical basis for seeking such a wave-particle model. For, consider 1-D 
Euler equations in primitive variables,

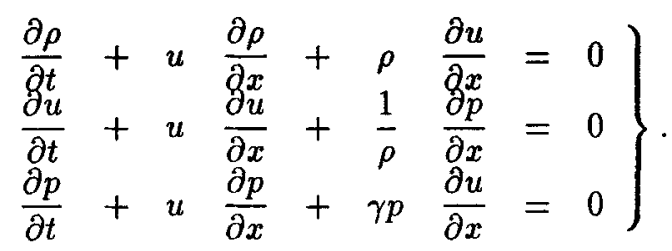

We have the famous convective derivative appearing in all the three equations and its appearance is due to the motion of fluid elements along particle paths. In order to demonstrate the wave nature let us write (4) in the matrix form.

$$
\frac{\partial V}{\partial t}+\tilde{A}^{t} \frac{\partial V}{\partial x}+\tilde{A}^{a} \frac{\partial V}{\partial x}=0
$$

where,

$$
V=\left[\begin{array}{l}
\rho \\
u \\
p
\end{array}\right] ; \tilde{A}^{t}=\left[\begin{array}{ccc}
u & 0 & 0 \\
0 & u & 0 \\
0 & 0 & u
\end{array}\right] ; \tilde{A}^{a}=\left[\begin{array}{ccc}
0 & \rho & 0 \\
0 & 0 & 1 / \rho \\
0 & \gamma p & 0
\end{array}\right]
$$

The eigenvalues of $\tilde{A}^{a}$ can be easily shown to be

$$
\lambda\left(\tilde{A}^{a}\right)=0, \pm a
$$

where $a=(\gamma p / \rho)^{1 / 2}=$ sonic speed. Thus the third term in (5) can be considered to represent propagation of acoustic waves in both directions in case of 1-D and all directions in case of multidimensional flows. We therefore take the view that the motion of fluid is a mixture of particle behaviour (the basis of Lagrangian description) and wave behaviour. This view is also supported by the fact that the rate of change of a conserved quantity (momentum or energy) in a control volume is due to the change caused by the transport of fluid (particle like) and the change caused by the pressure acting on the control volume. We can also regard the dynamics of fluid as consisting of the advection of the fluid element along particle paths and further these fluid elements are subjected to dilatation or contraction thus sending pressure waves into the domain. It is therefore physically meaningful to regard the fluid motion as partly particle-like and partly wave-like.

We have therefore a natural splitting of the flux vector into transport part and acoustic part and hence we write the 1-D Euler equation in the form,

$$
\frac{\partial U}{\partial t}+\frac{\partial F^{t}}{\partial x}+\frac{\partial F^{a}}{\partial x}=0
$$

where,

$$
F^{t}=\left[\begin{array}{l}
\rho u \\
\rho u^{2} \\
c u
\end{array}\right] ; F^{a}=\left[\begin{array}{l}
0 \\
p \\
p u
\end{array}\right]
$$

The corresponding flux Jacobians $A^{t}=\partial F^{t} / \partial U$ and $A^{a}=\partial F^{a} / \partial U$ are given by,

$$
A^{t}=\left[\begin{array}{ccc}
0 & 1 & 0 \\
-u^{2} & 2 u & 0 \\
-\frac{e u}{\rho} & \frac{e}{\rho} & u
\end{array}\right]
$$




$$
A^{a}=\left[\begin{array}{ccc}
0 & 0 & 0 \\
\frac{(\gamma-1)}{2} u^{2} & -(\gamma-1) u & (\gamma-1) \\
-\frac{p u}{\rho}+\frac{(\gamma-1)}{2} u^{3} & \frac{p}{\rho}-(\gamma-1) u^{2} & (\gamma-1) u
\end{array}\right]
$$

The eigenvalues of the matrices are given by

$$
\lambda\left(A^{t}\right)=u, u, u ; \lambda\left(A^{a}\right)=0, \pm[(\gamma-1) / \gamma]^{\frac{1}{2}} \quad a .
$$

Observe that the corresponding matrices $\tilde{A}^{t}$ and $\tilde{A}^{a}$ in the primitive variable representation have the eigenvalues $\lambda\left(\tilde{A}^{t}\right)=u, u, u$ and $\lambda\left(\tilde{A}^{a}\right)=0, \pm a$. Thus there is difference between $\lambda\left(\tilde{A}^{a}\right)$ and $\lambda\left(A^{a}\right)$ because of the transformation from $V$ representation to $U$-representation.

Now let us look at the construction of an upwind scheme for the Euler equations based on the wave-particle splitting idea. The term involving the flux $F^{t}$ does not pose any problem as far as implementing the upwinding is concerned. Based on whether $u$ is positive or negative $\partial F^{t} / \partial x$ can be backward or forward differenced for enforcing upwinding. The task of enforcing upwinding for the term $\partial F^{a} / \partial x$ is slightly more complex due to the mixed eigenvalues of $A^{a}$, that is eigenvalues are of mixed sign. Many variations of similar flux splitting exist but they are different from our wave-particle splitting. The Convective Upwind and Split Pressure (CUSP) schemes of Jameson (1993) split the flux as,

$$
F^{c}=\left[\begin{array}{l}
\rho u \\
\rho u^{2} \\
H u
\end{array}\right] ; F^{p}=\left[\begin{array}{l}
0 \\
p \\
0
\end{array}\right],
$$

where, $H=e+p$. These are different from $F^{t}$ and $F^{a}$ given by (9). Since the eigenvalues of $\partial F^{c} / \partial U$ are $u, u$ and $\gamma u$ while those of $\partial F^{p} / \partial U$ are 0,0 and $-(\gamma-1) u$, a splitting with,

$$
F^{+}=F^{c}, F^{-}=F^{p},
$$

leads to stable scheme as done by Denton (1983). As remarked by Jameson this scheme does not reflect the true zone of dependence in supersonic flow. He then modifies the scheme by an appropriate choice of diffusive flux. Again the Advective Upstream Splitting Method [AUSM] of Liou \& Steffen (1991) as different from the present wave-particle splitting in the sense that $F^{t}$ and $F^{p}$ chosen by them are not the same as the transport and acoustic fluxes given by (9). Conceptually we are using a physically meaningful basis for splitting $F$ into $F^{t}$ and $F^{a}$.

We (Balakrishnan \& Deshpande 1991,1992a,1994a,1994b, Balakrishnan and Raghurama Rao 1992b) have further split $A^{a}$ following a method similar to that of Steger \& Warming (1981). Here we apply this method to $F^{a}$ as against Steger \& Warming (1981) who considered the total flux $F$ instead. Towards this end we require the canonical form

$$
A^{a}=R^{a} \Lambda^{a}\left(R^{a}\right)^{-1}
$$

where $R^{a}=$ matrix of right eigenvectors of $A^{a}$ and $\Lambda^{a}=$ diagonal matrix and these are given by

$$
R^{a}=\left[\begin{array}{ccc}
0 & 1 & 0 \\
1 & u & 1 \\
u-\frac{a}{[\gamma(\gamma-1)]^{\frac{1}{2}}} & \frac{u^{2}}{2} & u+\frac{a}{[\gamma(\gamma-1)]^{\frac{1}{2}}}
\end{array}\right]
$$




$$
\Lambda^{a}=[(\gamma-1) / \gamma]^{\frac{1}{2}}\left[\begin{array}{ccc}
-a & 0 & 0 \\
0 & 0 & 0 \\
0 & 0 & a
\end{array}\right]
$$

We now split $\Lambda^{a}$ into $\Lambda^{a+}$ and $\Lambda^{a-}$ given by

$$
\Lambda^{a+}=[(\gamma-1) / \gamma]^{\frac{1}{2}}\left[\begin{array}{ccc}
0 & 0 & 0 \\
0 & \phi a & 0 \\
0 & 0 & a
\end{array}\right] ; \Lambda^{a-}=[(\gamma-1) / \gamma]^{\frac{1}{2}}\left[\begin{array}{ccc}
-a & 0 & 0 \\
0 & -\phi a & 0 \\
0 & 0 & 0
\end{array}\right]
$$

and then obtain the acoustic fluxes $F^{a \pm}$ defined by

$$
F^{a \pm}=R^{a} \Lambda^{a \pm}\left(R^{a}\right)^{-1}
$$

when mathematical manipulation in (17) are performed we obtain

$$
F^{a \pm}=\left[\begin{array}{llll} 
\pm[(\gamma-1) / \gamma]^{\frac{1}{2}} & \phi a & \rho & \\
\frac{p}{2} \pm[(\gamma-1) / \gamma]^{\frac{1}{2}} & \phi a & \rho u & \\
\frac{p u}{2} \pm \frac{p a}{2[\gamma(\gamma-1)]^{\frac{1}{2}}} \pm[(\gamma-1) / \gamma]^{\frac{1}{2}} & \phi a & \frac{\rho u^{2}}{2}
\end{array}\right]
$$

Here $\phi$ is a dissipation control parameter to be determined by requiring that the stability condition gives the best stability limit on the permissible time step. The variation of $\phi$ with Mach number is given by the following expression (Balakrishnan \& Deshpande 1994a):

$$
\phi= \begin{cases}1.37925-2.6774 M-4.34512 M^{2} & \text { for } \quad M \leq 0.234355 \\ 0.520381-0.030885 M & \text { for } \quad 0.234355<M \leq 10.373218 \\ 0.2 & \text { for } M>10.373218\end{cases}
$$

The above expression for $\phi$ are obtained by using a curve-fit for the stability plot. The linear stability analysis applied to the equation

$$
\frac{\partial}{\partial t^{\prime}}+A^{t} \frac{\partial U}{\partial x}+A^{a+} \frac{\partial U}{\partial x}+A^{a}-\frac{L U U}{\partial x}=0,
$$

is somewhat complex because the matrices $A^{t}, A^{a \pm}$ do not commute and hence do not admit simultaneous diagonalisation. Following the von Neumann stability analysis which assumes that,

$$
U^{n}(x)=\int \hat{U}^{n}(\xi) e^{2 \pi i \xi x} \mathrm{~d} \xi .
$$

We substitute for $U^{n}(x)$ in the upwind scheme,

$$
\frac{U_{k}^{n+1}-U_{k}^{n}}{\Delta t}+\frac{A_{k}^{t}\left(U_{k}^{n}-U_{k-1}^{n}\right)}{\Delta x}+\frac{A_{k}^{a+}\left(U_{k}^{n}-U_{k-1}^{n}\right)}{\Delta x}+{\frac{A_{k}^{a-}\left(U_{k+1}^{n}-U_{k}^{n}\right)}{\Delta x}}^{n}=0,
$$

for $u>0$ and,

$$
\frac{U_{k}^{n+1}-U_{k}^{n}}{\Delta t}+\frac{A_{k}^{t}\left(U_{k+1}^{n}-U_{k}^{n}\right)}{\Delta x}+\frac{A_{k}^{a+}\left(U_{k}^{n}-U_{k-1}^{n}\right)}{\Delta x}+\frac{A_{k}^{a-}\left(U_{k+1}^{n}-U_{k}^{n}\right)}{\Delta x}=0
$$


for $u<0$. We obtain the amplification matrix $G(\beta)$ in the form,

$$
G=I-\frac{\Delta t}{\Delta x} Y ; Y=E_{T} A^{t}+E_{B} A^{a+}+E_{F} A^{a-},
$$

where $E_{T}=E_{B}$ if $u>0$ and $E_{F}$ if $u<0$, and $E_{B}$ and $E_{F}$ are defined by

$$
E_{B}=1-e^{-i \beta}, E_{F}=e^{i \beta}-1, \beta=2 \pi \xi \Delta x .
$$

The spectral theory of stability demands that the spectral radius of $G$ be less than or equal to unity. Figure 1 shows the stability plots for the above scheme. These plots show the maximum allowed acoustic courant number $(a(\Delta t / \Delta x))$ as a function of Mach number for values of dissipation control parameter $\phi$ varying from 0 to 1.5. It is obvious that the Acoustic Flux Vector Splitting Scheme is unstable for very low Mach numbers when $\phi=0$. We can increase the low mach number limit of the allowed $a(\Delta t / \Delta x)$ by increasing $\phi$. Table 1 shows the real and imaginary parts of the eigenvalues of $A^{\text {at }}$.

Table 1. Eigenvalues of $A^{ \pm a}$ for various values of $\phi$

\begin{tabular}{ccc}
\multicolumn{3}{c}{ various values of $\phi$} \\
\hline \multirow{4}{*}{0.00} & \multicolumn{2}{c}{ Eigenvalues of $A^{ \pm a}$} \\
& Real parts & Imaginary parts \\
\hline & & \\
& & \\
& & \\
& \pm 0.000000 & 0.000000 \\
0.10 & \pm 0.0450000 & 0.000000 \\
& \pm 1.011320 & 0.000000 \\
& $\mp 0.158201$ & 0.000000 \\
& & 0.000000 \\
0.25 & \pm 0.090635 & 0.000000 \\
& \pm 1.034360 & 0.000000 \\
& & 0.000000 \\
0.50 & \pm 0.202128 & \pm 0.127213 \\
& \pm 0.202128 & $\mp 0.127213$ \\
& \pm 1.095740 & 0.000000 \\
& & \\
& \pm 0.455587 & \pm 0.269326 \\
1.00 & \pm 0.455587 & $\mp 0.269326$ \\
& \pm 1.338830 & 0.000000 \\
& & \\
& \pm 0.797879 & \pm 0.419213 \\
& \pm 0.797879 & $\mp 0.419213$ \\
& \pm 2.154240 & 0.000000 \\
\hline
\end{tabular}

A factor of $[(\gamma-1) / \gamma]^{\frac{1}{2}}$ has been pulled out of the eigenvalues in the above table for convenience. It is obvious from this table that for $\phi<0.25$ all the eigenvalues of 


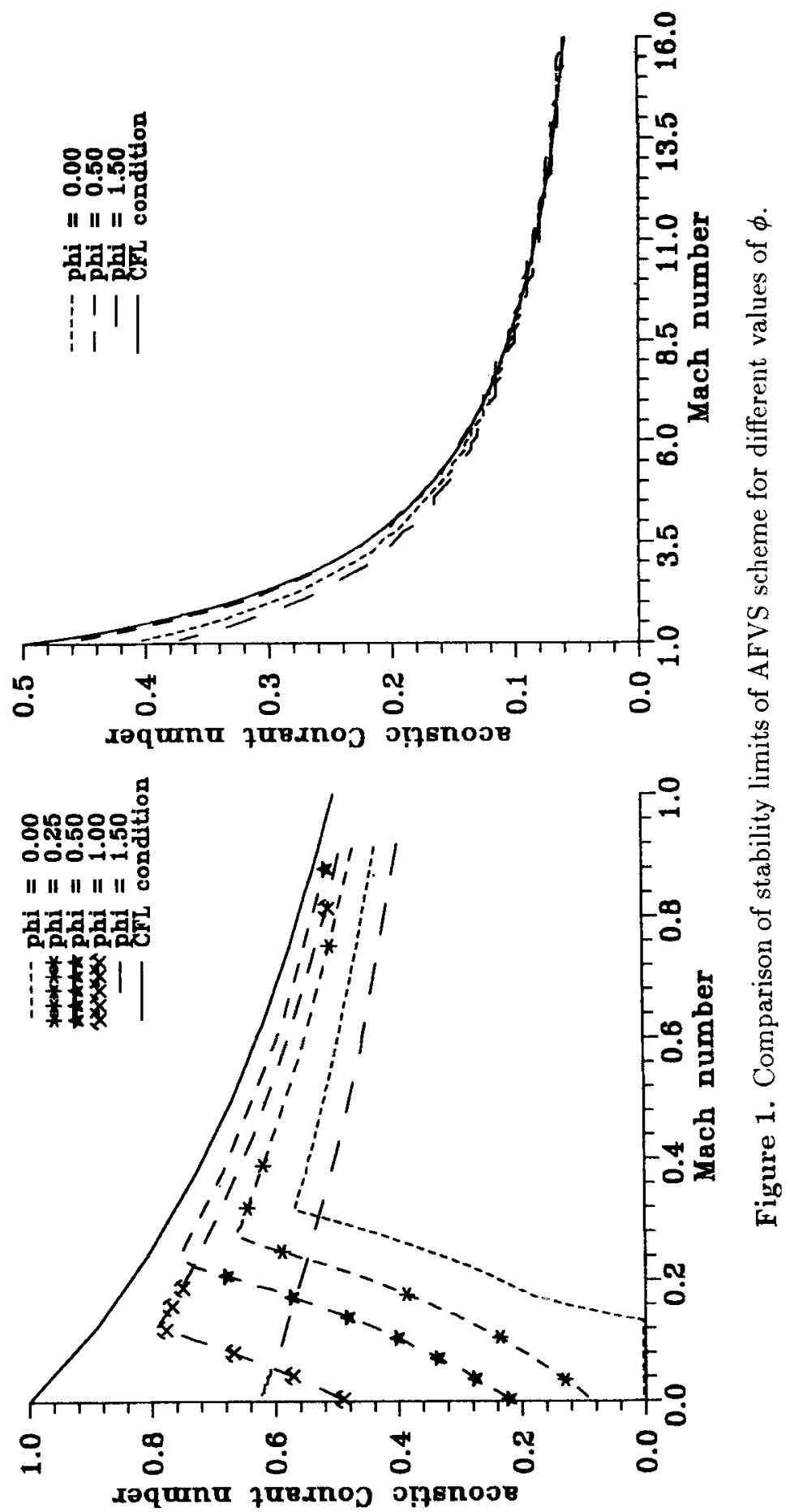


$A^{a+}$ and $A^{a-}$ are real but are of mixed sign. At $\phi=0.25$ all the eigenvalues of $A^{a+}$ are positive and those of $A^{a-}$ are negative. For values of $\phi>0.25$ the eigenvalues are complex. An interesting point that emerges from the present stability and eigenvalue analysis is that a stable upwind scheme based on split fluxes need not have all positive and all negative eigenvalues for the split flux Jacobians. This has been followed as an unnecessarily restrictive methodological principle in the past while designing FVS schemes.

\subsection{Acoustic flur difference splitting (AFDS)}

Acoustic flux difference splitting is yet another variant of the wave-particle splitting method. We start with the transport-acoustic split Euler equations (8),

$$
\frac{\partial U}{\partial t}+\frac{\partial F^{t}}{\partial x}+\frac{\partial F^{a}}{\partial x}=0
$$

as before but treat the term $\partial F^{a} / \partial x$ differently (Balakrishnan and Deshpande $1991 \mathrm{a}, 1992)$. The transport term $\partial F^{t} / \partial x$ is upwind differenced as before. The acoustic term $\partial F^{a} / \partial x$ is discretised by using Flux Difference Splitting. There are many ways of achieving this discretisation. In the AFDS version studied here we use Roc-linearisation. We must hasten to add that Roe (1981) has treated the unsplit fux $F$ in this manner while we use Roe's approach for the acoustic flux vector $F^{a}$ only. For this purpose following Ror, we introduce the parameter vector,

$$
w_{1}=\sqrt{\rho} ; \quad w_{2}=\sqrt{\rho} u ; w_{3}=p / \sqrt{\rho},
$$

which is somewhat different from the parameter vector used by Roe. In terms of the above parameter vector the conserved vector $U$ and acoustic flux vector $F^{a}$ become

$$
U=\left[\begin{array}{l}
w_{1}^{2} \\
w_{1} w_{2} \\
\frac{w_{1} w_{3}}{(\gamma-1)}+\frac{w_{2}^{2}}{2}
\end{array}\right] \text { and } F^{a}=\left[\begin{array}{l}
0 \\
w_{1} w_{2} \\
w_{2} w_{3}
\end{array}\right]
$$

The corresponding averaged quantities are defined by,

$$
\left.\begin{array}{rl}
\bar{u} & =\frac{\bar{w}_{2}}{\bar{w}_{1}}=\frac{\sqrt{\rho_{L}} u_{L}+\sqrt{\rho_{R}} u_{R}}{\sqrt{\rho_{L}}+\sqrt{\rho_{R}}} \\
\bar{a}^{\prime} & =\frac{\gamma \bar{w}_{3}}{\bar{w}_{1}}=\frac{\gamma\left(p_{L} / \sqrt{\rho_{L}}+p_{R} / \sqrt{\rho_{R}}\right)}{\sqrt{\rho_{L}}+\sqrt{\rho_{R}}}
\end{array}\right\} .
$$

The averaged matrix $\ddot{A}^{a}$ is given by,

$$
\bar{A}^{a}=\left[\begin{array}{ccc}
0 & 0 & 0 \\
\frac{(\gamma-1)}{2} \bar{u}^{2} & -(\gamma-1) \bar{u} & (\gamma-1) \\
-\frac{\bar{a}^{2} \bar{u}}{\gamma}+\frac{(\gamma-1)}{2} \bar{u}^{3} & \frac{\bar{a}^{2}}{\gamma}-(\gamma-1) \bar{u}^{2} & (\gamma-1) \bar{u}
\end{array}\right]
$$

It is interesting to observe that averaged $\ddot{A}^{a}$ inatrix is very similar to $A^{a}$ given by (11) except that $u$ and $a$ in (11) are replaced by $\ddot{u}$ and $a$ defined above. The matrix $\bar{A}^{a}$ satisfies the well-known property,

$$
\Delta F=\bar{A}^{a} \Delta U,
$$


where $\Delta F^{a}=F_{R}^{a}-F_{L}^{a}, \Delta U=U_{R}-U_{L}$. Because of the similarity between $\bar{A}^{a}$ and $A^{a}$ it follows that,

$$
\bar{\lambda}\left(\bar{A}^{a}\right)=0, \pm \bar{a}[(\gamma-1) / \gamma]^{\frac{1}{2}} .
$$

The corresponding right eigenvectors are,

$$
\mathbf{r}_{1}=\left[\begin{array}{l}
0 \\
1 \\
\bar{u}-\frac{\bar{a}}{[\gamma(\gamma-1)]^{\frac{1}{2}}}
\end{array}\right] ; \mathbf{r}_{2}=\left[\begin{array}{l}
1 \\
\bar{u} \\
\frac{\bar{u}^{2}}{2}
\end{array}\right] ; \mathbf{r}_{3}=\left[\begin{array}{l}
0 \\
1 \\
\bar{u}+\frac{\vec{a}}{[\gamma(\gamma-1)]^{\frac{1}{2}}}
\end{array}\right] .
$$

The acoustic flux $F_{C}^{a}$ at the cell interface are calculated using the formula

$$
F_{C}^{a}=\frac{1}{2}\left[\left(F_{L}^{a}+F_{R}^{a}\right)-\sum_{j=1}^{3} \Delta \psi_{j}^{a} \vec{r}_{j}\left|\lambda_{j}\right|\right]
$$

where $L$ and $R$ stand for the states to the left and right of the cell face $C$ and $\Delta \psi_{j}$ are given by,

$$
\left.\begin{array}{rl}
\Delta \psi_{1}^{a} & =\frac{\bar{\rho} \Delta u}{2}-\sqrt{\gamma /(\gamma-1)} \frac{\Delta p}{2 \bar{a}} \\
\Delta \psi_{2}^{a} & =\Delta \rho \\
\Delta \psi_{3}^{a} & =\frac{\bar{\rho} \Delta u}{2}+\sqrt{\gamma /(\gamma-1)} \frac{\Delta p}{2 \bar{a}}
\end{array}\right\}
$$

and,

$$
\Delta \rho=\rho_{R}-\rho_{L}, \Delta u=u_{R}-u_{L}, \Delta p=p_{R}-p_{L}
$$

The total flux at a cell face $C$ is then given by the sum of upwind interpolated transport flux $F^{t}$ and $F_{C}^{a}$. As a last comment on AFDS we may add that even though AFDS is based on our wave-particle idea it is somewhat different from AFVS described before. Both AFDS and AFVS treat the transport part of the flux in the same fashion but acoustic part $F^{a}$ on a cell face is computed differently by these methods.

\section{Peculiar velocity based upwind (PVU) method}

Here we discuss yet another line of approach for construction of novel algorithms for Euler equations of gas dynamics. This line of attack called the PVU method, is an application of the moment method strategy so successfully applied by Deshpande (1986c) and Mandal (1989) and Mandal \& Deshpande (1994) to develop the Kinetic Flux Vector Splitting (KFVS) method. The KFVS method, which has been recently surveyed by Deshpande (1993), has turned out to be extremely robust in numerically solving a variety of $2-\mathrm{D}$ and $3-\mathrm{D}$ problems arising in inviscid gas dynamics. The KFVS method suffers from three basic deficiencies :

(i) like many other flux vector splitting methods it is highly diffusive. This property is both a virtue and a vice. The KFVS method owes its robustness to its highly diffusive character (virtue) and ipso facto leads to unacceptably large smearing of contact discontinuity and boundary layers (vice) ;

(ii)the KFVS method assumes a rest frame because the splitting is accomplished by dividing the molecular velocity into positive half $(v>0)$ and negative half $(v<0)$; (iii) the integration of the Maxwellian distribution over $v>0$ and $v<0$ in KFVS 
leads to formulae involving error functions whose computation for every mesh point can be expensive.

Raghurama Rao \& Deshpande (1991a,1995) have recently advanced the concept of splitting based on the peculiar velocity $c$ (also called the thermal velocity in the jargon of the Kinetic Theory of Gases). The split flux expressions so obtained are free of the defects (ii) and (iii) above, and lead to the PVU method which is more efficient than the KFVS method while at the same time possessing its robust property.

We will now describe the basis of the PVU method (Raghurama Rao \& Deshpande (1991a,1995)). Consider the 1-D Boltzmann equation

$$
\frac{\partial f}{\partial t}+v \frac{\partial f}{\partial x}=J(f, f)
$$

where $v$ is the molecular velocity, $f$ is the velocity distribution function, $J(f, f)$ is the collision term whose structure is of no concern here as it vanishes in the Euler limit. The basic unknown $f$ in (35) is a function of time $t$, position $x$ and velocity $v$. The Maxwellian velocity distribution denoted by $F$ is given by

$$
F=\frac{\rho}{I_{0}}(\beta / \pi)^{\frac{1}{2}} \exp \left[-\beta(v-u)^{2}-\frac{I}{I_{0}}\right],
$$

where $\rho$ is the mass density, $\beta=\frac{1}{2 R T}, T$ is the temperature, $R$ is the gas constant per unit mass, $u$ is the fluid velocity, $I$ is the internal energy variable corresponding to nontranslational degrees of freedom (this variable is required to force a given value of $\gamma$ for the gas), $I_{0}$ is the average internal energy due to nontranslational degrees of freedom, given by $I_{0}=[(3-2 \gamma) /(2(\gamma-1))] R T$ and $\gamma$ is the ratio of specific heats. One of important properties of

$$
\frac{\partial F}{\partial t}+v \frac{\partial F}{\partial x}=0
$$

is that the Euler equations of motion,

$$
\frac{\partial U}{\partial t}+\frac{\partial G}{\partial x}=0
$$

can be cast in the compact form

$$
\left\langle\Psi, \frac{\partial F}{\partial t}+v \frac{\partial F}{\partial x}\right\rangle=0
$$

where

$$
\begin{aligned}
& \Psi=\text { moment function vector }=\left[\begin{array}{c}
1 \\
v \\
I+\frac{v^{2}}{2}
\end{array}\right], \\
& U=\langle\Psi, F\rangle=\int_{0}^{\infty} \mathrm{d} I \int_{-\infty}^{\infty} \mathrm{d} v \Psi F=\left[\begin{array}{c}
\rho \\
\rho u \\
\rho E
\end{array}\right],
\end{aligned}
$$




$$
G=\langle\Psi, F\rangle=\int_{0}^{\infty} \mathrm{d} I \int_{-\infty}^{\infty} \mathrm{d} v \Psi v F=\left[\begin{array}{c}
\rho u \\
p+\rho u^{2} \\
p u+\rho u E
\end{array}\right]
$$

The above connection between the Boltzmann equation (37) and the Euler equations (38) is at the root of many kinetic schemes. Raghurama Rao \& Deshpande (1991a,1995) rewrite (37) in the form,

$$
\frac{\partial F}{\partial t}+\frac{\partial}{\partial x}(u F)+\frac{\partial}{\partial x}(c F)=0
$$

where $c=v-u$ is the peculiar velocity. Taking $\Psi$-moments of (43) gives

$$
\frac{\partial U}{\partial t}+\frac{\partial G^{t}}{\partial x}+\frac{\partial G^{a}}{\partial x}=0
$$

where

$$
\begin{aligned}
& G^{t}=\langle\Psi, u F\rangle=\int_{0}^{\infty} \mathrm{d} I \int_{-\infty}^{\infty} \mathrm{d} c \Psi u F, \\
& G^{a}=\langle\Psi, c F\rangle=\int_{0}^{\infty} \mathrm{d} I \int_{-\infty}^{\infty} \mathrm{d} c \Psi c F .
\end{aligned}
$$

The flux vectors $G^{t}$ and $G^{a}$ defined by (44) and (45) are exactly the same as $F^{t}$ and $F^{a}$ given by (9). It is interesting to observe that the physical arguments leading to the equation (8) involving splitting of the flux vector into the transport and the acoustic parts, are different from those behind equation (44). Even then both ways of looking at the splitting of the flux vector lead to identical expressions for the transport and the acoustic parts. The basic idea behind writing the Boltzmann equation (39) in the form given by (45) is the recognition that the $u$-part is unidirectional while the c-part is multidirectional. Another way of looking at this difference is that $u$ is a deterministic variable while the random variable $c \sim N(0,1 /(2 \beta))$, that is, $c$ follows a Normal distribution with zero mean and variance equal to $1 /(2 \beta)$. The motion of a particle can be thought of as consisting of an orderly motion $(u)$ and a random motion $(\epsilon)$ due to thermal agitation of molecules. The PVU method recognises this difference in the behaviour of $u$-term and $c$-term and treats them differently. It is also believed that this way of dealing with the two terms is useful in constructing genuinely multidimensional upwind schernes.

The next question in the development of the PVU method is the upwind differencing of $\partial G^{t} / \partial x$ and $\partial G^{a} / \partial x$ terms. So far as the transport term is concerned we follow the same method as before, that is, we write

$$
\frac{\partial}{\partial x}(u F)=\frac{\partial}{\partial x}\left(\frac{u+|u|}{2}\right)+\frac{\partial}{\partial x}\left(\frac{u-|u|}{2}\right),
$$

and then obtain the upwind differenced approximation as

$$
\begin{aligned}
{\left[\frac{\partial}{\partial x}(u F)\right]_{j} } & =\frac{1}{\Delta x}\left[\left(\frac{u+|u|}{2} F\right)_{j}-\left(\frac{u+|u|}{2} F\right)_{j-1}\right] \\
& +\frac{1}{\Delta x}\left[\left(\frac{u-|u|}{2} F\right)_{j+1}-\left(\frac{u-|u|}{2} F\right)_{j}\right] .
\end{aligned}
$$


After taking $\Psi$-moments as per the moment method strategy (Deshpande 1986c) we obtain the upwind differencing at the Euler level as

$$
\begin{aligned}
\left(\frac{\partial G^{t}}{\partial x}\right)=\left[\frac{\partial}{\partial x}(u F)\right]_{j} & =\frac{1}{\Delta x}\left[\left(\frac{u+|u|}{2} F\right)_{j}-\left(\frac{u+|u|}{2} F\right)_{j-1}\right] \\
& +\frac{1}{\Delta x}\left[\left(\frac{u-|u|}{2} F\right)_{j+1}-\left(\frac{u-|u|}{2} F\right)_{j}\right] .
\end{aligned}
$$

Let us now deal with the upwind differencing of the acoustic term $\partial G^{a} / \partial x$. Following Raghurama Rao \& Deshpande (1991a,1995) we observe that

$$
\begin{aligned}
\frac{\partial G^{a}}{\partial x} & =\left\langle\Psi, \frac{\partial}{\partial x}(c F)\right\rangle \\
& =\left\langle\Psi, \frac{\partial}{\partial x}\left(\frac{c+|c|}{2} F\right)\right\rangle+\left\langle\Psi, \frac{\partial}{\partial x}\left(\frac{c-|c|}{2} F\right)\right\rangle \\
& =\frac{\partial}{\partial x}\left\langle\Psi, \frac{c+|c|}{2} F\right\rangle+\frac{\partial}{\partial x}\left\langle\Psi, \frac{c-|c|}{2} F\right\rangle
\end{aligned}
$$

We therefore obtain split acoustic fluxes $G^{a+}$ and $G^{a-}$ given by

$$
G^{a+}=\left\langle\Psi, \frac{c+\mid c}{2} F\right\rangle \text { and } G^{a-}=\left\langle\Psi, \frac{c-\mid c}{2} F\right\rangle
$$

This splitting is based on the peculiar velocity $c$ and hence this scheme is called Peculiar Velocity based Upwind (PVU) scheme. In terms of the split fluxes (48) gives

$$
\frac{\partial G^{a}}{\partial x}=\frac{\partial G^{a+}}{\partial x}+\frac{\partial G^{a-}}{\partial x} .
$$

Performing the integration with respect to $c$ and $I$ in the formulae (49) we obtain

$$
G^{a \pm}=\left[\begin{array}{c} 
\pm \frac{\rho}{2 \sqrt{\pi \beta}} \\
\frac{p}{2} \pm \frac{\rho u}{2 \sqrt{\pi \beta}} \\
\frac{p u}{2} \pm \frac{1}{2 \sqrt{\pi \beta}}\left(\frac{p}{2}+\rho E\right)
\end{array}\right]
$$

Both the AFVS and PVU schemes are somewhat similar to the CUSP (Convection Upwind and Split Pressure) scheme of Jameson (1993) in the sense that the convection terms in the Euler equations are upwind differenced and the pressure terms are split. However, the CUSP scheme is different from the present methods. The transport flux and the acoustic flux of AFVS and PVU methods are not the same as the convective flux and pressure flux either in the CUSP scheme of Jameson or that due to Denton (1983). Hence, the eigenvalues of the corresponding flux Jacobians are also different for CUSP and the present methods. The method of upwind differencing the convective and pressure fluxes is also different in the AFVS and PVU methods compared to the CUSP method. Let us now compare the expressions for the split acoustic fluxes for the AFVS and PVU methods. We note that $\beta=\gamma /\left(2 a^{2}\right)$ 
and hence $G^{a \pm}$ for the PVU method reduce to

$$
G^{a \pm}=\left[\begin{array}{c} 
\pm \frac{\rho a}{\sqrt{2 \pi \gamma}} \\
\frac{p}{2} \pm \frac{\rho u a}{\sqrt{2 \pi \gamma}} \\
\frac{p u}{2} \pm \frac{\rho a}{\sqrt{2 \pi \gamma}}\left(\frac{p}{\rho} \frac{\gamma+1}{2(\gamma-1)}+\frac{u^{2}}{2}\right)
\end{array}\right]
$$

Now compare these with the AFVS expression (18)

$$
G^{a \pm}=\left[\begin{array}{c} 
\pm \sqrt{\frac{\gamma-1}{\gamma}} \phi \rho a \\
\frac{p}{2} \pm \sqrt{\frac{\gamma-1}{\gamma}} \phi \rho u a \\
\frac{p u}{2} \pm \rho a \sqrt{\frac{\gamma-1}{\gamma}}\left(\frac{p}{\rho(\gamma-1)}+\frac{\phi u^{2}}{2}\right)
\end{array}\right]
$$

We observe that PVU formulae for $G^{a \pm}$ given by (52) are very similar to those of AFVS given by (18). However, they are not identical even if we try to choose a suitable value of the dissipation control parameter $\phi$ in (18). For, a comparison between (18) and (52) suggests that

$$
\phi=\frac{1}{[2 \pi(\gamma-1)]^{\frac{1}{2}}} .
$$

This value when substituted in expressions (18) yields

$$
G^{a \pm}=\left[\begin{array}{c} 
\pm \frac{\rho a}{\sqrt{2 \pi \gamma}} \\
\frac{p}{2} \pm \frac{\rho u a}{\sqrt{2 \pi \gamma}} \\
\frac{p u}{2} \pm \frac{\rho a}{\sqrt{2 \pi \gamma}}\left(\frac{p}{\rho} \frac{2 \pi}{\gamma-1}+\frac{u^{2}}{2}\right)
\end{array}\right]
$$

which shows that the split fluxes for mass and momentum are in agreement with the respective expressions for the PVU method but the split fluxes for energy do not match. We therefore conclude that the AFVS and PV-splitting are allied ideas but they do not lead to identical expressions for the split fluxes even when the parameter $\phi$ is adjusted. It appears that one more parameter may be required for obtaining identical formulae. Whether this is possible within the framework of AFVS remains to be seen.

Finally we end this section by stating that the PV-splitting can be easily extended to multidimensions. We start with the 2-D Boltzmann equation

$$
\frac{\partial F}{\partial t}+\frac{\partial}{\partial x}\left(v_{1} F\right)+\frac{\partial}{\partial x}\left(v_{2} F\right)=0
$$

The collision term gets dropped as it becomes zero in the Euler limit. The 2-D Maxwellian $F$ is given by

$$
F=\frac{\rho}{I_{0}} \frac{\beta}{\pi} \exp \left[-\beta\left(v_{1}-u_{1}\right)^{2}-\beta\left(v_{1}-u_{1}\right)^{2}-\frac{I}{I_{0}}\right]
$$




$$
I_{0}=\frac{2-\gamma}{\gamma-1} R T \text {. }
$$

Following the same procedure as before we rewrite (62) in the form

$$
\frac{\partial F}{\partial t}+\frac{\partial}{\partial x}\left(u_{1} F\right)+\frac{\partial}{\partial x}\left(u_{2} F\right)+\frac{\partial}{\partial x}\left(c_{1} F\right)+\frac{\partial}{\partial x}\left(c_{2} F\right)=0
$$

and define the transport and acoustic fluxes by

$$
G_{1}^{t}=\left\langle\Psi, u_{1} F\right\rangle, G_{2}^{t}=\left\langle\Psi, u_{2} F\right\rangle, G_{1}^{a}=\left\langle\Psi, c_{1} F\right\rangle, G_{2}^{a}=\left\langle\Psi, c_{2} F\right\rangle,
$$

The split acoustic fluxes are then given by

$$
G_{1}^{a \pm}=\left\langle\Psi, \frac{c_{1} \pm\left|c_{1}\right|}{2} F\right\rangle, G_{2}^{a \pm}=\left\langle\Psi, \frac{c_{2} \pm\left|c_{2}\right|}{2} F\right\rangle
$$

Performing the integrations in the formulae (60) with respect to $I, c_{1}$ and $c_{2}$ we obtain

$$
\begin{gathered}
G_{1}^{a \pm}=\left[\begin{array}{c} 
\pm \frac{\rho}{2 \sqrt{\pi \beta}} \\
\frac{p}{2} \pm \frac{\rho u_{1}}{2 \sqrt{\pi \beta}} \\
\pm \frac{\rho u_{2}}{2 \sqrt{\pi \beta}} \\
G_{2}^{a \pm}=\left[\begin{array}{c}
\left.\frac{p+1}{\rho} \frac{\gamma+1)}{2(\gamma-1)}+\frac{u_{1}^{2}+u_{2}^{2}}{2}\right)
\end{array}\right] \\
\pm \frac{\rho}{2 \sqrt{\pi \beta}} \\
\pm \frac{\rho u_{1}}{2 \sqrt{\pi \beta}} \\
\frac{p}{2 \sqrt{\pi \beta}} \pm \frac{\rho u_{2}}{2 \sqrt{\pi \beta}} \\
\left(\frac{p}{\rho} \frac{\gamma+1}{2(\gamma-1)}+\frac{u_{1}^{2}+u_{2}^{2}}{2}\right)
\end{array}\right]
\end{gathered}
$$

The above formulae are based on splitting along $x-y$ directions of a Cartesian frame. Wher PVU is used in the finite volume framework, the fluxes must be determined on a cell face not necessarily parallel to either of the coordinate directions. On an arbitrary cell face the split fluxes are given by

$$
G^{a \pm}=\left[\begin{array}{c} 
\pm \frac{\rho}{2 \sqrt{\pi \beta}} \\
n_{1} \frac{p}{2} \pm \frac{\rho u_{1}}{2 \sqrt{\pi \beta}} \\
n_{2} \frac{p}{2} \pm \frac{p u_{1}}{2 \sqrt{\pi \beta}} \\
\frac{p u_{n}}{2} \pm \frac{\rho}{2 \sqrt{\pi \beta}}\left(\frac{p}{2 \rho}+E\right)
\end{array}\right]
$$

where $n_{1}$ and $n_{2}$ are the direction cosines of the outward normal on the cell face, $u_{n}$ is the fluid velocity normal to the coll face and $E$ is the internal energy per unit mass. We observe that the split flux formuale ( ${ }^{a \pm}$ of the PVU method do not 
involve error functions and exponential terms and hence are expected to lead to a more efficient computational method than the KFVS method. Also, no rest frame is assumed in deriving the above formulae. The next section discusses the results obtained by solving a large number of 2-D problems using AFVS, AFDS and PVU methods.

\section{Results and discussions}

For some preliminary results obtained using the AFDS method reference is made to Balakrishnan \& Deshpande (1991). AFDS method has been found to be less dissipative and less robust than the AFVS method. Here we concentrate on the results obtained by using AFVS and PVU methods.

Both the AFVS and PVU schemes have been tested on a large number of 2D problems (Raghurama Rao(1994) and Balakrishnan and Deshpande (1992). It is a standard practice in Computational Fluid Dynamics to evaluate the performance of new schemes by computing subsonic, transonic and supersonic flows for GAMM/AGARD test cases. We give below a few examples demonstrating the capability of these two new methods. More details are available in Raghurama Rao (1994) and Balakrishnan \& Deshpande (1992). The AFVS method was applied to compute flow past NACA0012 airfoil on $128 \times 64 \mathrm{O}$ - type structured grid with outer boundary five chord lengths away from the mid-chord point. The free stream Mach number is 0.85 and the angle of attack is $1^{\circ}$. This is an AG $\Lambda$ RD test case. The pressure and Mach contours obtained are shown in figure 2. The contours are smooth and are indicative of the ability of the scheme to capture shocks accurately.

The PVU method was also applied to the above test example. The computations were done on an unstructured mesh with adaptation. High resolution finite volume version of PVU method was used (Raghurama Rao 1994). The starting mesh, the adapted mesh as well as the pressure contours obtained on these meshes are shown in figures $3,4,5$ and 6 . Only a part of the mesh is shown for clarity. The outer boundary is located 15 chord lengths away from the airfoil, which is not shown in the above figures. The shocks on the upper and the lower surfaces are very accurately captured. Table 2 shows the lift and drag coefficients $\left(C_{L}\right.$ and $\left.C_{D}\right)$ obtained by AFVS and PVU schemes. Also shown in this table are the standard values of these coefficients in the AGARD report (AGARD 1986).

Table 2. Lift and drag coefficients obtained with AFVF and PVU schemes.

\begin{tabular}{ccccccc} 
Case & \multicolumn{2}{c}{ A(iARD range } & \multicolumn{2}{c}{ AFVS } & \multicolumn{2}{c}{ PVU } \\
\hline$M_{\infty} \alpha$ & $C_{L}$ & $C_{D}$ & $C_{L}$ & $C_{D}$ & $C_{L}$ & $C_{D}$ \\
\hline $0.851^{0}$ & $0.330-0.3889$ & $0.0464-0.0590$ & 0.4145 & 0.0607 & 0.3351 & 0.0565 \\
\hline
\end{tabular}

The coefficients predicted by more accurate computations using PVU method fall within the AGARD range. The AFVS computations were not done with mesh adaptation and the coefficients predicted by the AFVS method are slightly outside the AGARD range. More accurate computations done by using AFVS method push these values into the AGARD range.

At the outer limit of the speed range is the hypersonic flow involving blunt body shock. The performance of the AFVS scheme was further tested by computing 


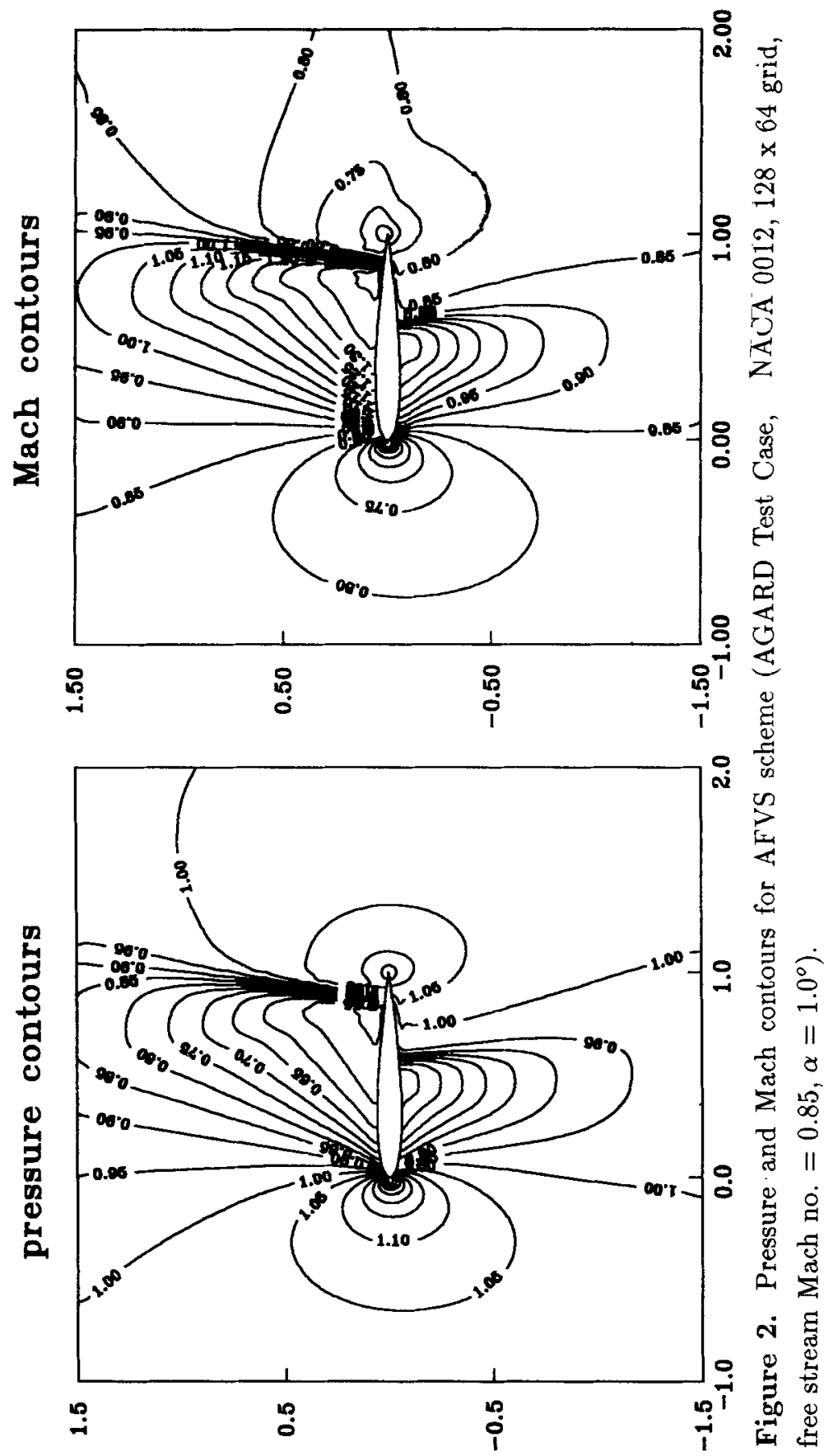




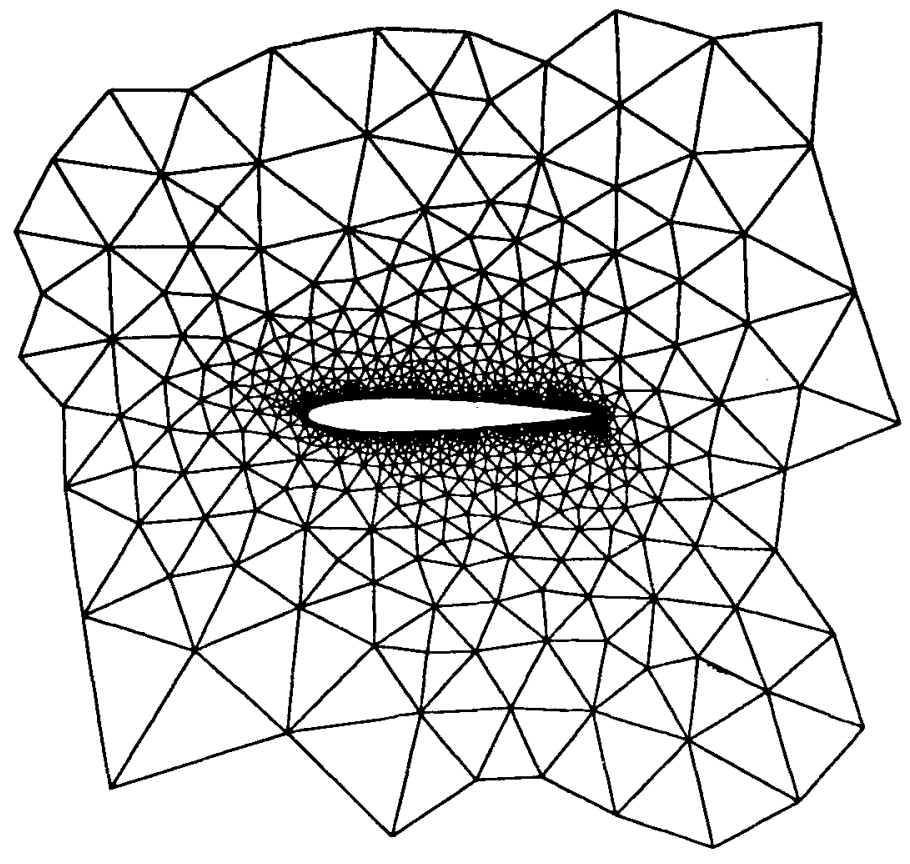

Figure 3. Starting mesh for NACA0012 airfoil, points $=1123$, cells $=2056$, edges $=3179$.

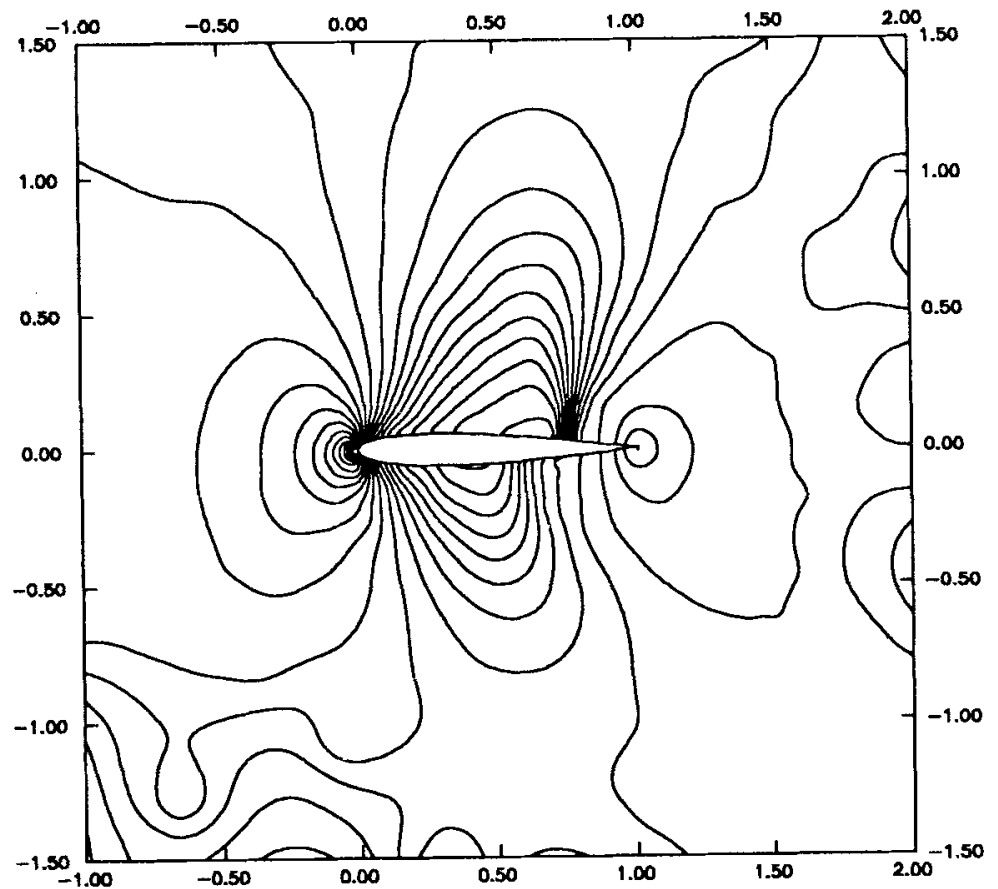

Figure 4. Pressure contours obtained on the starting mesh. Contour levels from 0.54 to 1.5 (increment 0.04 ), scheme: High resolution PVU finite volume method, Mach no. $=0.85$, angle of attack $=1^{\circ}$. 


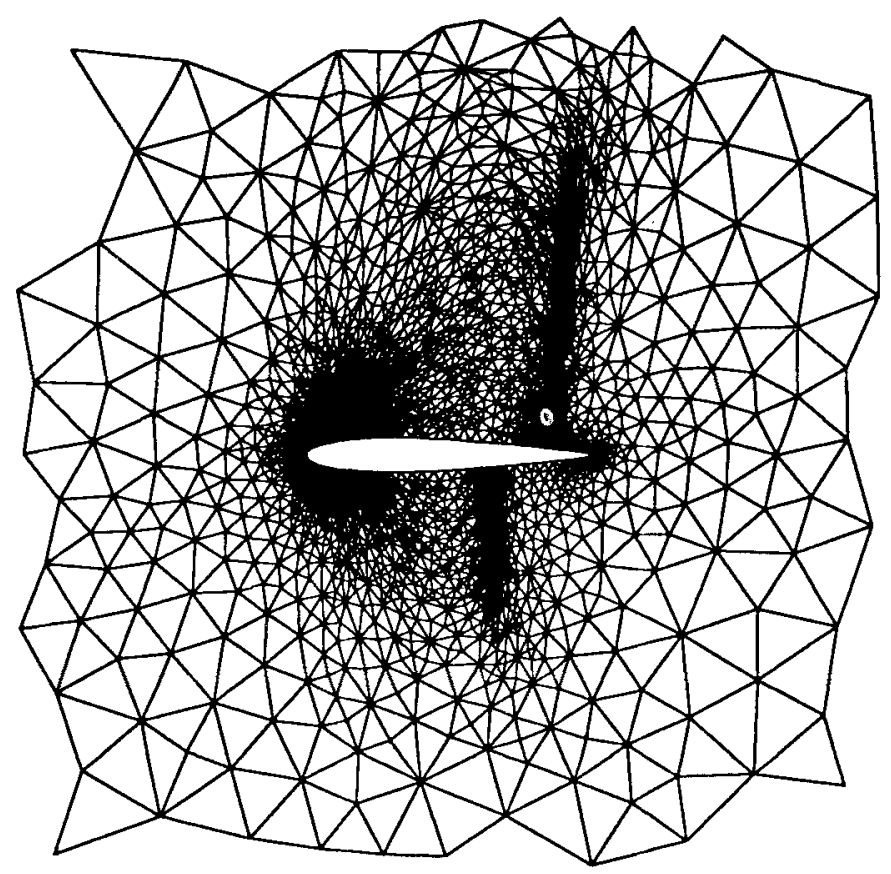

Figure 5. Adapted mesh for NACA0012 airfoil, points $=5724$, cells $=11197$, edges $=16921$.

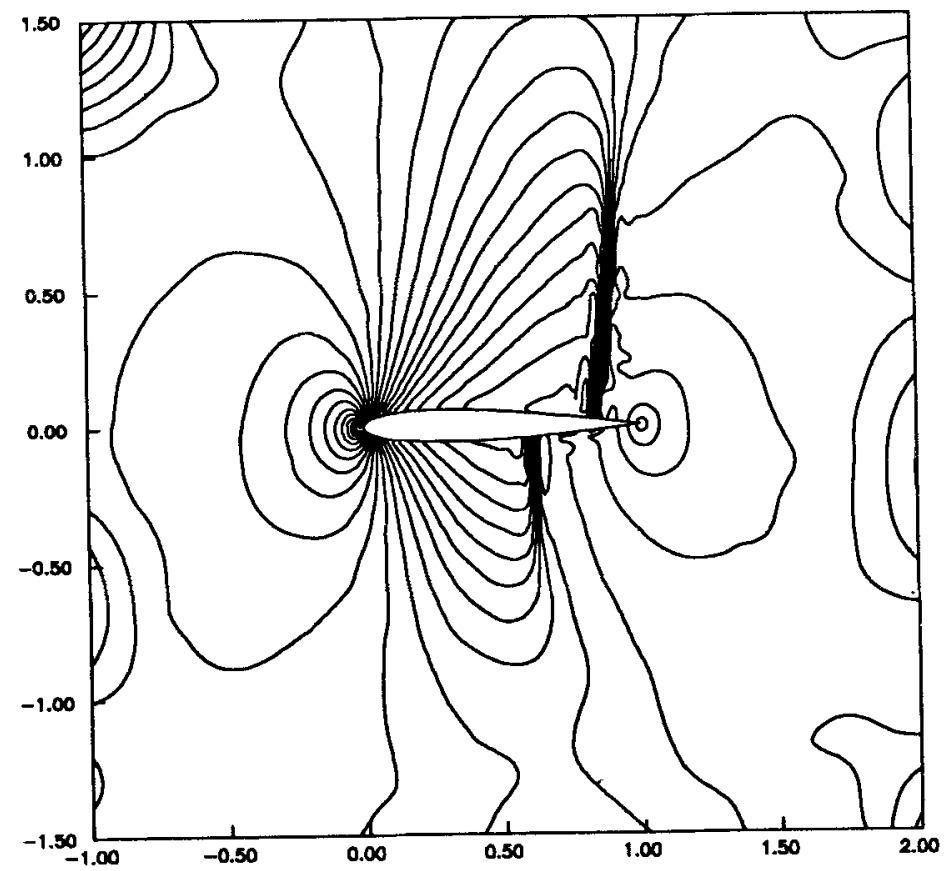

Figure 6. Pressure contours obtained on the adapted mesh. Contour levels from 0.48 to 1.5 (increment 0.04), scheme: High resolution PVU finite volume method, Mach no. $=0.85$, angle of attack $=1^{\circ}$. 
Mach no. $=4.0$

Mach no. $=10.0$

Mach no. $=15.0$
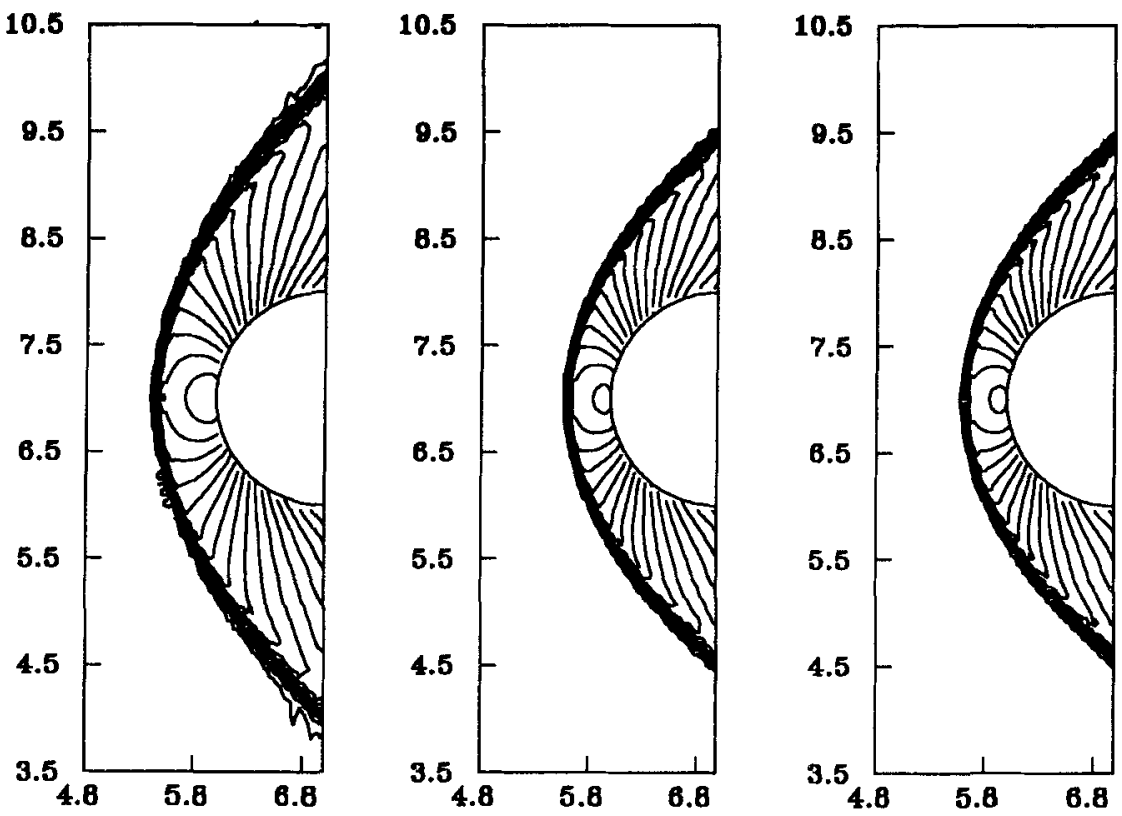

Figure 7. Pressure contours for AFVS scheme (flow past cylinder, 99 x 98 grid, MUSCL, contours 0.05 units apart).

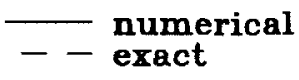

Mach no. $=4.0$

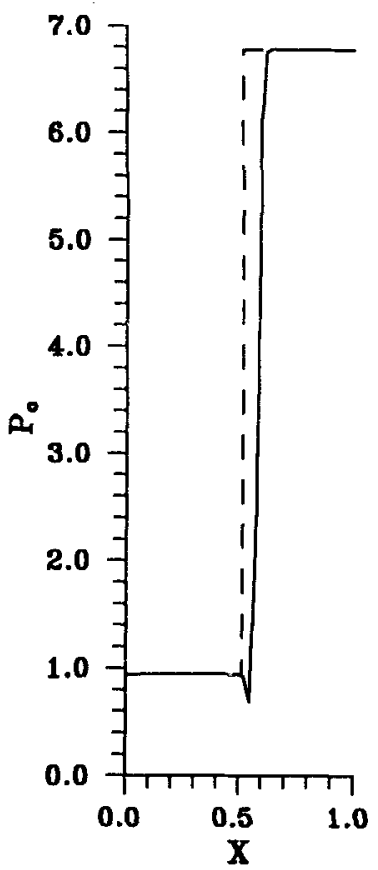

Mach no. $=10.0$

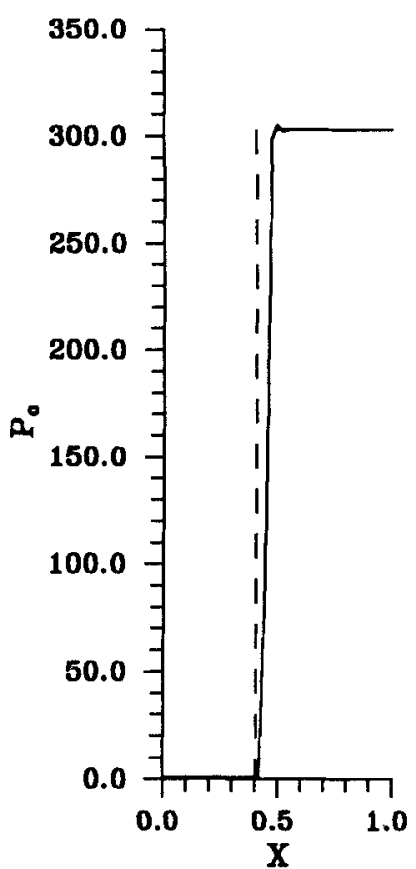

Mach no. $=15.0$

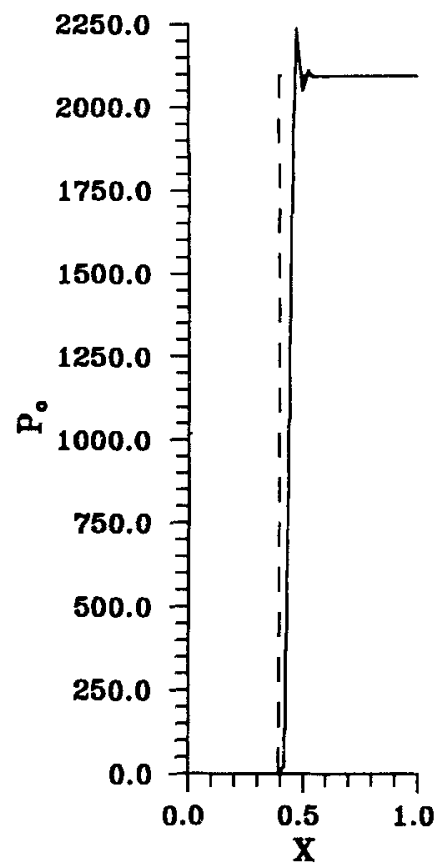

Figure 8. $P_{o}$ variation along the stagnation line for AFVS scheme (flow past a cylinder, 99 × 98 grid, MUSCL). 

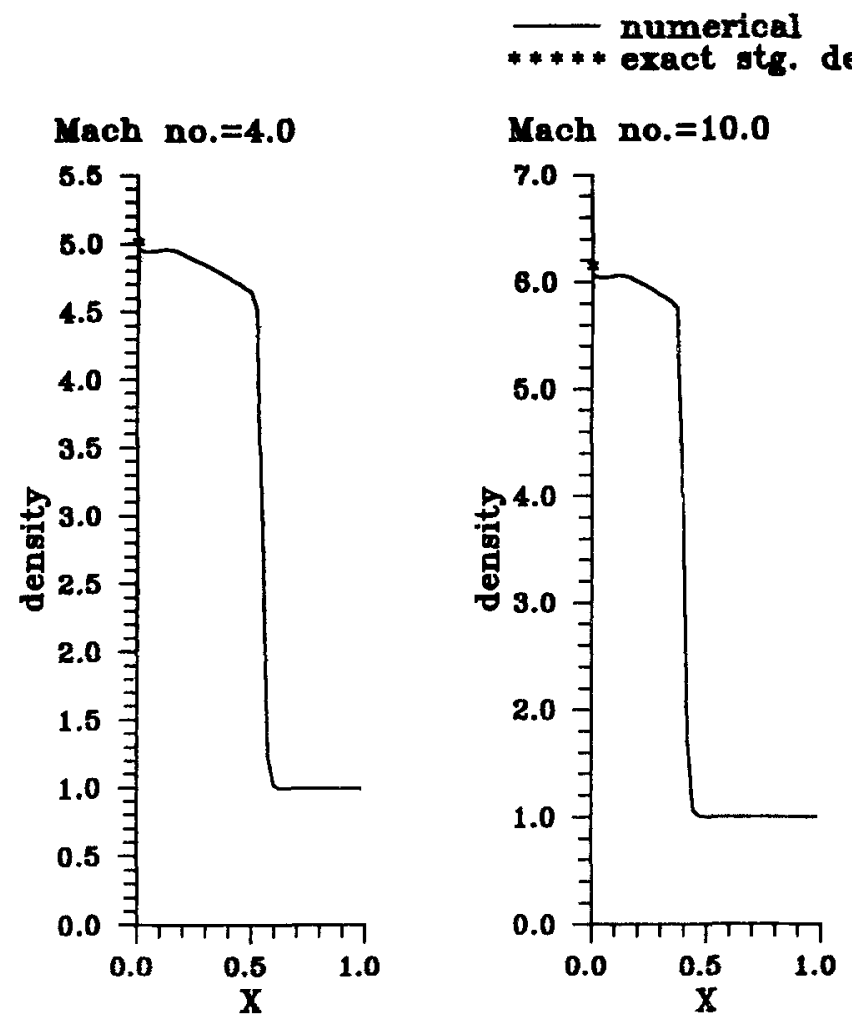

Figure 9. Density variation along the stagnation line for AFVS scheme (flow past a cylinder, $99 \times 98$ grid, MUSCL).

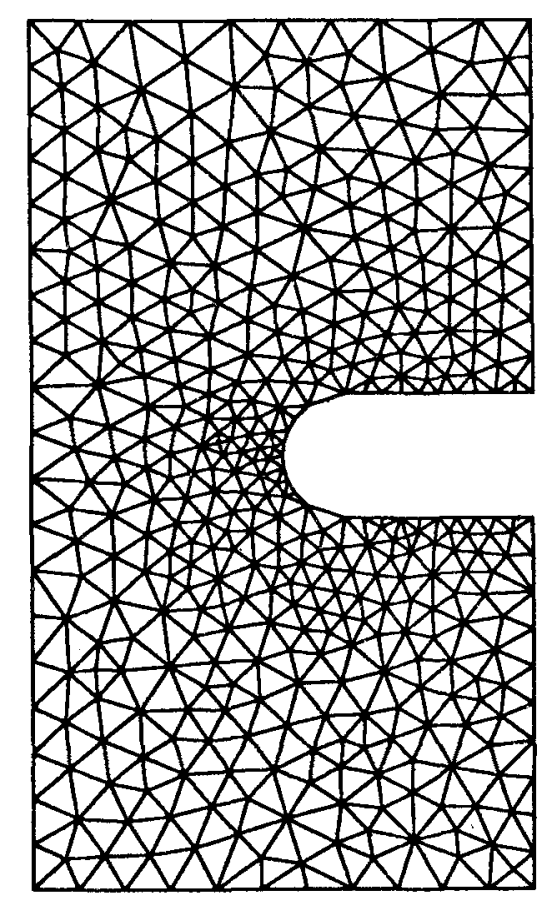

Figure 10. Starting mesh for blunt body, points $=446$, cells $=800$, edges $=1245$. 

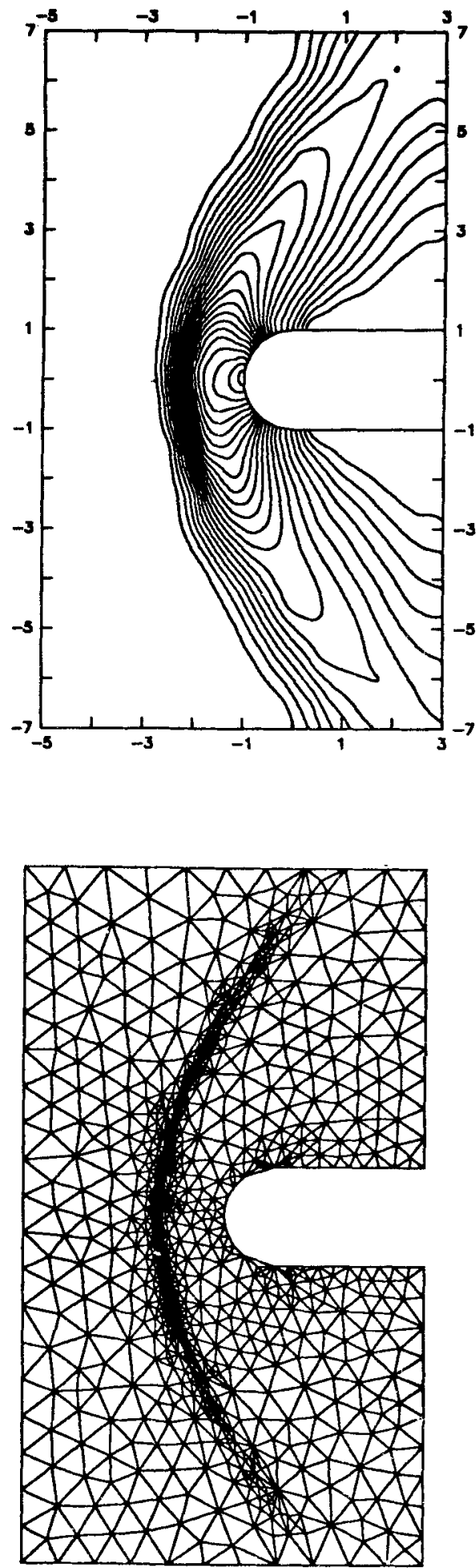

Figure 11. Pressure contours on starting mesh. Contour levels from 1.2 to 5.5 (increment 0.2 ), Mach no. $=2.0$, angle of attack $=0^{\circ}$, scheme: High resolution PVU finite volume method.
Figure 12. Adapted mesh for supersonic flow, points $=1017$, cells $=1942$, edges $=2958$. 

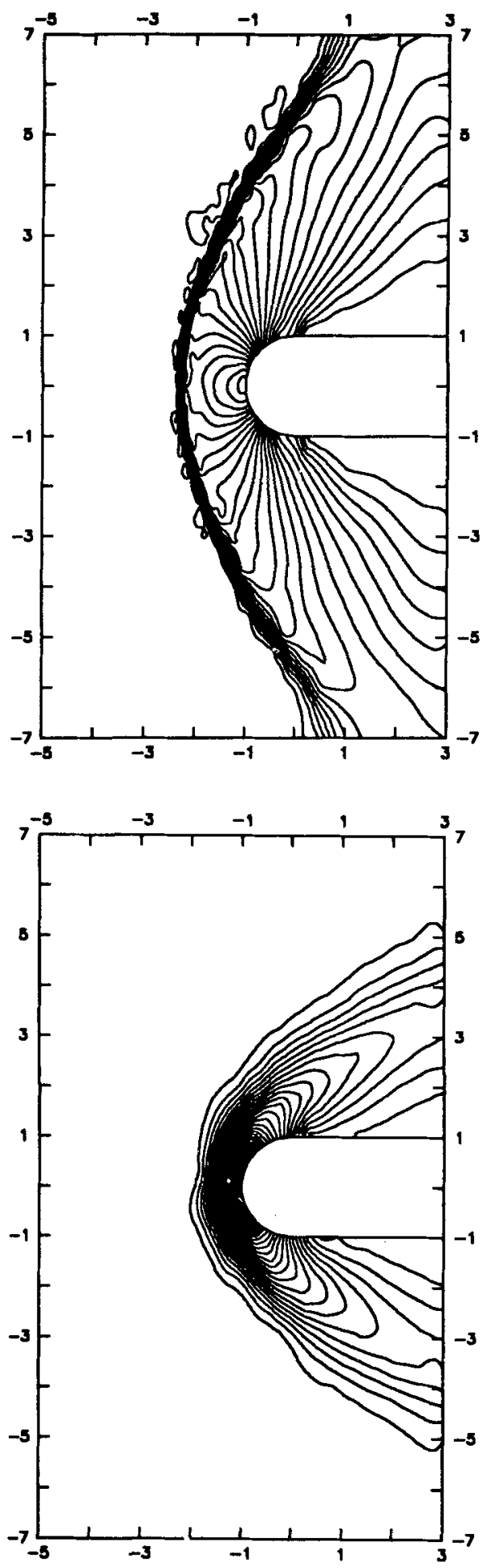

Figure 13. Pressure contours on adapted mesh. Contour levels from 0.7 to 5.7 (increment 0.2 ), Mach no. $=2.0$, angle of attack $=0^{\circ}$, scheme: High resolution PVU finite volume method.

Figure 14. Pressure contours on starting mesh. Contour levels from 2.0 to 78.0 (increment 2.0), Mach no. $=8.0$, angle of attack $=0^{\circ}$, scheme: First order PVU finite volume method. 

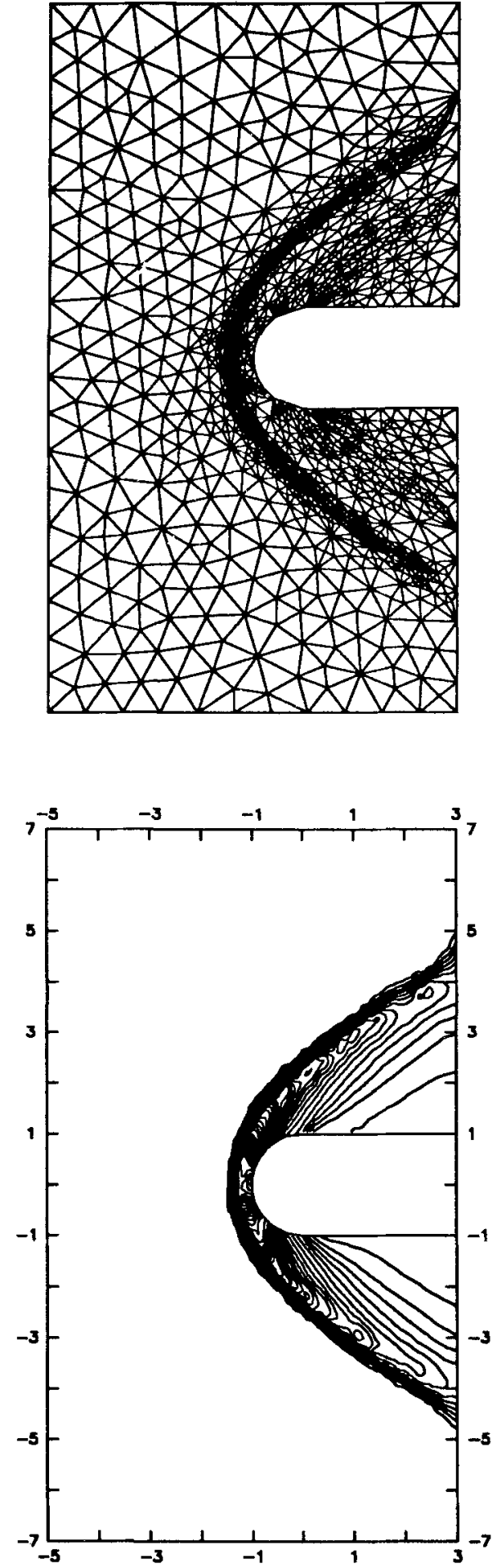

Figure 15. Adapted mesh for hypersonic flow, points $=1520$, cells $=2940$, edges $=4459$.

Figure 16. Pressure contours on adapted mesh. Contour levels from 2.0 to 82.0 (increment 2.0), Mach no. $=8.0$, angle of attack $=0^{\circ}$, scheme: First order PVU finite volume method. 
flow past a semi-cylinder. The computations were made for three different different mach numbers : 4, 10 and 15. A uniform $99 \times 98$ structured grid is employed for computations. The radius of the outer boundary is chosen to be 3.5 times the radius of the semi-cylinder. The pressure contours given by the AFVS scheme for three different Mach numbers, the total pressure variation and the density variation along the stagnation line are shown in figures 7,8 and 9 . The smoothness of these contours demonstrate the robustness of the AFVS scheme in capturing strong shocks.

The PVU scheme was applied to the test problem given by Arminjon \& Dervieux (1993) for hypersonic flow. The computations are done for $M=2.0$ and $M=8.0$ with zero angle of attack using high resolution and first order PVU scheme respectively on an unstructured mesh. Again the starting mesh, adapted mesh and the pressure contours obtained on these meshes are shown in figures 10 to 16 . The bow shock is closer to the body in hypersonic case compared to the supersonic flow. This is a well-known result of gas dynamics. The shocks are captured crisply by the PVU method with grid adaptaion.

Extensive computations performed using the AFVS and PVU schemes show the basic soundness of the wave-particle idea and splitting based on the peculiar velocity of molecules. The basic idea of having a discrete mathematical model inheriting as many physical properties of the fluid flow as possible appears quite sound and turns out to be often promising needing further study. Implicit in the AFVS method is the physically meaningful model that fluid behaves partly particle like and partly wave like. The PVU method is based on a different but closely related idea that the motion of particles is a random motion superimposed on a unidirectional motion. The particle like behaviour in wave-particle splitting is equivalent to the unidirectional motion in the PVU method. The random motion taking place in all directions is similar to the wave spreading in all directions. It would be very tempting to design a new scheme taking the multidirectionality of the pressure part into account. The genuinely multidimensional upwind scheme based on the Boltzmann equation has been developed by Raghurama Rao \& Deshpande (1991b) and Eppard \& Grossman (1993). The results show a lot of improvernent but these schemes are at present quite expensive. These two schemes also do not separate unidirectional and random motion of the molecules as in the PVU scheme. Further work is required to exploit these ideas in a more efficient manner.

\section{References}

AGARD 1986 Test cases for inviscid flow field methods. Report of Fluid Dynamics Panel Working Group 07, AGARD-AR 211

Arminjon P, Dervieux A 1993 Construction of TVD-like artificial viscosities in two-dimensional arbitrary FEM grids. J. Comput. Phys. 106: 176-198

Balakrishnan N, Deshpande S M 1991 New upwind schemes with wave-particle splitting for inviscid compressible flows. 91 FM 11, Fluid Mechanics Report, Dept. Aerosp. Eng., Indian Institute of Science, Bangalore

Balakrishnan N, Deshpande S M 1992a Assessment of the performance of waveparticle splitting with other flux vector and flux difference splitting schemes. 92 FM 7, Fluid Mechanics Report, Dept. Aerosp. Eng., Indian Institute of 
Science, Bangalore (Also presented in IMACS symp. held in Bangalore, India, December-1992)

Balakrishnan N, Deshpande S M 1994a New upwinding scheme based on the waveparticle splitting Computational fluid dynamics, '94. Proc. Second European CFD Conference. (eds) S Wagner, E H Hirschel, J Periaux, R Piva (Chichester: John Wiley \& Sons) pp 1-8

Balakrishnan N, Deshpande S M 1995 New upwind method exploiting the waveparticle behaviour of fluid flow.Comput. Fluid Dyn. J. (in press)

Balakrishnan N, Raghurama Rao S V 1992b New flux vector and flux difference splitting methods for Euler equations of gas dynamics. Proc. 5th Asian Cong. on Fluid Mechanics Taejon, Korea

Denton J D 1983 An improved time-marching method for turbomachinery flow calculations. J. Eng. Gas Turbines Power 105

Deshpande S M 1986a On the Maxwellian distribution, symmetric form and entropy conservation for Euler equations. NASA-TP-2583

Deshpande S M 1986b Kinetic theory based new upwind methods for inviscid compressible flows. AIAA Paper 86-0275

Deshpande S M 1993 Boltzmann schemes for continum gas dynamics. Sadhana 18: 405-430

Eppard W M, Grossman B 1993 A Multidimensional kinetic-based upwind solver for Euler equations. AIAA Paper No. 93-3303 CP

Jameson A 1993 Artificial diffusion, upwind biasing, limiters and their effect on accuracy and multigrid convergence in transonic and hypersonic flows. AIAA Paper 93-3359

Jameson A, Schmidt W, Turkel E 1981 Numerical solution of Euler equations finite volume method using Runge Kutta time stepping. AIAA-81-1259

Liou M S, Steffen J Jr 1991 A new flux-splitting scheme. NASA T'M 104404, NASA Lewis Research Centre, Cleveland, Ohio

MacCormack R W 1990 Solution of the Navier-Stokes equation in three dimensions. AIAA Paper No. 90-1520

Mandal J C 1989 Kinetic upwind method for inviscid compressible flows. Ph D thesis, Dept. Aerosp. Eng., Indian Institute of Science, Bangalore

Mandal J C, Deshpande S M 1994 Kinetic flux vector splitting for Euler equations. Comput. Fluids 23: 447-478

Osher S, Solomon F 1982 Upwind difference schemes for hyperbolic system of conservation laws. Math. Comput. 38: 339

Quirk J J 1992 A contribution to the great Reimann solver debate. ICASE Report NO. $92-64$ 
Raghurama Rao S V 1994 New upwind methods based on kinetic theory for inviscid compressible flows. Ph D thesis, Dept. Mech. Eng., Indian Institute of Science, Bangalore

Raghurama Rao S V, Deshpande S M 1991a A class of efficient kinetic upwind methods for compressible flows. 91 FM 12, Fluid Mechanics report, Dept. Aerosp. Eng., Indian Institute of Science, Bangalore

Raghurama Rao S V, Deshpande S M 1991b A genuinely multidimensional ypwind Boltzmann scheme for Euler equations. 91 FM 6, Fluid Mechanics Report, Dept. Aerosp. Eng., Indian Institute of Science, Bangalore

Raghurama Rao S V, Deshpande S M 1994 Peculiar velocity based upwind method on unstructured meshes. Computational fluid dynamics '94. Proc. Second European CFD Conference. (eds) S Wagner, E H Hirschel, J Periaux, R Piva (Chichester: John Wiley \& Sons) pp. 89 96

Raghurama Rao S V, Deshpande S M 1995 Peculiar velocity based upwind method for inviscid compressible flows. Comput. Fluid Dyn. J., Jpn, (to appear)

Roe P L 1981 Aproximate Reimann solvers, parameter vectors and difference schemes. J. Comput. Phy. 43: 357-372

Sanders R H, Prendergast K H 1974 The possible relation of the 3-kilospace arm to the explosions in the galactic nucleus. Astrophys. J. 188: 489-500

Steger J L, Warming R F 1981 F'ux vector splitting of inviscid gas dynamic equations with application to finite difference methods. J. Comput. Phys. 40: 263-293

van Leer B 1982 Flux vector splitting for Euler equations. Lecture Notes in Physics 170: 507

van Leer B 1990 Flux vector splitting for 1990's. Invited Lecture for the CFD Symposium on Aeropropulsion, NASA Lewis Research Center, NACA CP3078 